\title{
Growth hormone modulates hippocampal excitatory synaptic transmission and plasticity in old rats
}

\author{
Doris P. Molina ${ }^{\mathrm{a}}$, Olusegun J. Ariwodola ${ }^{\mathrm{b}}$, Constance Linville ${ }^{\mathrm{a}}$, William E. Sonntag ${ }^{\mathrm{b}, \mathrm{c}}$, \\ Jeff L. Weiner ${ }^{\mathrm{b}}$, Judy K. Brunso-Bechtold ${ }^{\mathrm{a}, \mathrm{d}}$, Michelle M. Adams ${ }^{\mathrm{a}, \mathrm{d}, \mathrm{e}, *}$ \\ ${ }^{a}$ Departments of Neurobiology and Anatomy, Wake Forest University School of Medicine, Winston-Salem, NC, USA \\ ${ }^{b}$ Department of Physiology and Pharmacology, Wake Forest University School of Medicine, Winston-Salem, NC, USA \\ ${ }^{c}$ Department of Geriatric Medicine, University of Oklahoma Health Science Center, Oklahoma City, OK, USA \\ ${ }^{d}$ Roena Kulynych Center for Memory and Cognition Research, Wake Forest University School of Medicine, Winston-Salem, NC, USA \\ e Department of Psychology, Bilkent University, Bilkent, Ankara, Turkey
}

Received 19 April 2011; received in revised form 10 August 2011; accepted 10 September 2011

\begin{abstract}
Alterations in the alpha-amino-3-hydroxy-5-methyl-4-isoxazolepropionate receptor (AMPA-R) and N-methyl-D-aspartate receptor (NMDA-R) have been documented in aged animals and may contribute to changes in hippocampal-dependent memory. Growth hormone $(\mathrm{GH})$ regulates AMPA-R and NMDA-R-dependent excitatory transmission and decreases with age. Chronic GH treatment mitigates age-related cognitive decline. An in vitro CA1 hippocampal slice preparation was used to compare hippocampal excitatory transmission and plasticity in old animals treated for 6-8 months with either saline or GH. Our findings indicate that GH treatment restores NMDA-Rdependent basal synaptic transmission in old rats to young adult levels and enhances both AMPA-R-dependent basal synaptic transmission and long-term potentiation. These alterations in synaptic function occurred in the absence of changes in presynaptic function, as measured by paired-pulse ratios, the total protein levels of AMPA-R and NMDA-R subunits or in plasma or hippocampal levels of insulin-like growth factor-I. These data suggest a direct role for GH in altering age-related changes in excitatory transmission and provide a possible cellular mechanism through which GH changes the course of cognitive decline.
\end{abstract}

(C) 2012 Elsevier Inc. All rights reserved.

Keywords: Long-term potentiation; Paired-pulse ratios; Input-output curves; AMPA receptor; NMDA receptor

\section{Introduction}

Growth hormone $(\mathrm{GH})$ plays an important role in the central nervous system throughout the lifespan. GH is secreted by the anterior pituitary and is involved in neural processes including neurogenesis, gliogenesis, cell migration, and differentiation, synaptogenesis, and myelination, as well as neuroprotection (Aberg, 2010; Aberg et al., 2006, Aberg et al., 2010; Isgaard et al., 2007). GH levels are high during development and puberty, and then decline with

\footnotetext{
* Corresponding author at: Psychology Department, Bilkent University, 06800 Bilkent, Ankara, Turkey. Tel.: +90(8) 312290 1090; fax: +90(8) 3122902561 .

E-mail address: michelle@bilkent.edu.tr (M.M. Adams).
}

advancing age (Sonntag et al., 1980). Correlated with this decline is a reduction in cognitive function in rodents, demonstrated by an age-related impairment in performance on the Morris water maze (MWM), a hippocampal-dependent test of spatial learning and memory (Fischer et al., 1989; Frick et al., 1995; Olton and Werz, 1978; Rapp et al., 1987; Rosenzweig et al., 1997). Interestingly, old rats receiving 4 to 8 months of GH treatment perform significantly better on the MWM than age-matched, untreated controls (Ramsey et al., 2004), suggesting that GH can mitigate the age-related changes in hippocampal function that underlie this cognitive impairment.

The GH-induced mitigation of impaired hippocampal function may occur as a result of modulating glutamatergic synaptic transmission in the hippocampus. Glutamate is the 
primary excitatory neurotransmitter in the hippocampus and glutamatergic synaptic transmission involves the activation of ionotropic receptors which include the $\alpha$-amino-3hydroxy-5-methyl-4-isoxazole propionate (AMPA)-and Nmethyl-D-aspartate (NMDA)-types of glutamate receptors (Hollmann and Heinemann, 1994). The hippocampal levels of these receptors are related to performance on the MWM task (Adams et al., 2001; Tsien et al., 1996) and they mediate long-term potentiation (LTP), a process thought to underlie learning and memory (Bliss and Lomo, 1973; Morris et al., 1986). Importantly, changes in the subunits that comprise AMPA and NMDA receptors (AMPA-R and NMDA-R) have a significant impact on basal synaptic transmission as well as LTP (Cao et al., 2007; Granger et al., 1996; Lynch et al., 2006; Tang et al., 1999; Tsien et al., 1996) and there are age-related changes in basal synaptic transmission (Barnes et al., 1997; Eckles-Smith et al., 2000), LTP (Clayton et al., 2002; Eckles-Smith et al., 2000; Woodruff-Pak et al., 2010) and the protein levels of individual subunits have been reported to decline with age (Adams et al., 2008; Magnusson, 1998; Newton et al., 2008; Shi et al., 2006, 2007; Sonntag et al., 2000). Finally, in old rats, $\mathrm{GH}$ has been found to regulate the levels of the NMDA-R subunit transcript levels (Le Grevès et al., 2002). Thus, GH may ameliorate hippocampal-dependent cognitive decline by regulating AMPA-Rs and NMDA-Rs.

It is known that insulin-like growth factor-I (IGF-I) can serve as an anabolic mediator of the biological effects of GH. IGF-I has been shown to alter short-term presynaptic facilitation (Ramsey et al., 2004), AMPA-R-mediated synaptic transmission (Ramsey et al., 2005; Wang and Linden, 2000), and LTP (Trejo et al., 2007). Nevertheless, GH itself has been shown to directly modulate glutamatergic synaptic transmission in the CA1 region of hippocampus. Specifically, acute GH application enhanced compound and pharmacologically isolated NMDA-R and AMPA-R field excitatory postsynaptic potentials (fEPSPs) in hippocampal slices not only from young rats (Mahmoud and Grover, 2006), but also from old rats (Molina et al., unpublished observations) at an age when the $\mathrm{GH}$-induced amelioration of cognitive impairment has been shown to occur (Ramsey et al., 2004). These reports, together with the presence of GH receptor mRNA in the brain (Burton et al., 1992; Fraser et al., 1990; Hull and Harvey, 1998; Lobie et al., 1993, 2000; Walsh et al., 1990; Zhai et al., 1994) and evidence that GH crosses the blood brain barrier (Pan et al., 2005), suggest that GH may directly influence glutamate receptor function. The direct positive modulatory effect of $\mathrm{GH}$ on excitatory synaptic transmission in the hippocampus provides a potential mechanism for the $\mathrm{GH}$-induced amelioration of cognitive function in aged rats.

Although acute application of GH can directly enhance synaptic transmission in hippocampal slices from old rats, no studies to date have determined if the regimen of chronic $\mathrm{GH}$ treatment that has been shown to mitigate age-related cognitive impairment in old rats also results in the enhancement of glutamatergic synaptic function in old rats. To address this issue, we have compared basal excitatory synaptic transmission and LTP in the hippocampal CA 1 region of old rats treated with $\mathrm{GH}$ for 6-8 months with that in young adult and old saline-treated rats. Our results indicate that 6-8 months of GH treatment restores NMDA-R-dependent basal glutamatergic synaptic transmission in CA1 region in old rats to young adult levels and enhances both AMPA-R-dependent basal glutamatergic synaptic transmission and LTP in the CA1 region in old rats above the levels in young adult rats, but does not alter presynaptic release, as measured by paired-pulse ratios. These effects of chronic $\mathrm{GH}$ treatment occur in the absence of significant changes in overall tissue levels of NMDA and AMPA glutamate receptor subunits, or increases in plasma or hippocampal levels of IGF-I.

\section{Methods}

\subsection{Animals}

Young (9-11 months; $n=33$ ) and old (29-31 months; $n=76)$ male Fisher $344 \times$ Brown Norway $(F 344 \mathrm{X} \mathrm{BN})$ rats were obtained from the National Institute of Aging Colony at Harlan Sprague Dawley. One half of the old rats were subcutaneously injected with $300 \mu \mathrm{g}$ of porcine $\mathrm{GH}$ twice daily for 6-8 months (O-GH). All animals were treated at the same time and 36 animals were used for the protein and IGF-I analyses and the remaining 73 were used for the electrophysiological studies. The dosage and regimen employed have been demonstrated to result in improved MWM function in old rats (Ramsey et al., 2004). The other half of the old rats and all of the young rats were injected with saline (O-S and Y-S, respectively) for the same time period. Body weights were recorded every 2 weeks for the duration of the study. The animal protocol for this study conforms to National Institute of Health guidelines for the care and use of laboratory animals and was approved by the Animal Care and Use Committee of Wake Forest University Health Sciences.

\subsection{Drug preparation}

Porcine GH (Ozbiopharm, Knoxfield, Australia) was prepared as a stock solution in deionized water. For the pharmacological isolation of AMPA-R-dependent fEPSPs the drug, DL-2-amino-5-phosphonovaleric acid (APV; Sigma, St. Louis, MO, USA; $20 \mu \mathrm{M}$ ), a selective NMDA receptor blocker was used. To isolate the NMDA-R-dependent fEPSPs, 6,7-dinitroquinoxaline-2,3-dione (DNQX, Sigma, St. Louis, MO, 50 $\mu \mathrm{M}$ ), a selective AMPA/kainic acid (KA) receptor blocker, combined with bicuculline methylbromide (BIC; Sigma; 20 $\mu \mathrm{M})$ a $\gamma$-aminobutyric acid type $\mathrm{A}\left(\mathrm{GABA}_{\mathrm{A}}\right)$ channel blocker, and glycine (Sigma; $1 \mu \mathrm{M}$ ), an allosteric modulator of NMDA-Rs were used. The drugs were prepared as stock solutions in dimethyl sulfoxide (DMSO; Sigma; final concentra- 
tion $<0.05 \%$ ) for DNQX or in deionized water for APV and bicuculline methylbromide.

\subsection{Hippocampal slice preparation}

Rats to be used for hippocampal slice recordings were anesthetized with halothane (Sigma) and decapitated with a guillotine. The brains were removed and submerged in chilled, oxygenated artificial cerebrospinal fluid (aCSF) containing $124 \mathrm{mM} \mathrm{NaCl}, 3.3 \mathrm{mM} \mathrm{KCl}, 2.4 \mathrm{mM} \mathrm{MgCl}_{2}, 2.5$ $\mathrm{mM} \mathrm{Ca} \mathrm{Cl} 2,1.2 \mathrm{mM} \mathrm{KH}_{2} \mathrm{PO}_{4}, 10-\mathrm{D}$-glucose, and $25.9 \mathrm{mM}$

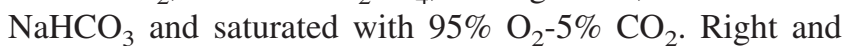
left hemispheres were separated and cut coronally into $400-\mu \mathrm{m}$ thick slices using a vibratome (Leica VT1000S; Vashaw Scientific, Atlanta, GA, USA). The hippocampus in each slice was dissected from surrounding tissue, and equilibrated in an incubation chamber with oxygenated aCSF at $20-25{ }^{\circ} \mathrm{C}$ for 90 minutes. During experiments, slices were placed in a recording chamber and perfused with oxygenated aCSF at a flow rate of $2 \mathrm{~mL} /$ minute at $32{ }^{\circ} \mathrm{C}$.

\subsection{Electrophysiology}

Basal synaptic transmission, paired-pulse ratios and LTP were studied by eliciting fEPSPs in hippocampal slices. Recording electrodes were prepared from filamented borosilicate glass capillary tubes $(0.86 \mathrm{~mm}$ inner diameter $)$ using a horizontal micropipette puller (Sutter P-97; Sutter, Novato, CA, USA) and filled with aCSF. For all recording experiments, after slices were transferred to a recording chamber, a twisted bipolar stimulating electrode (FHC, Bowdoinham, ME, USA) was placed in the Schaffer collaterals of area the CA3 region and fEPSPs were recorded from the stratum radiatum of the CA1 region (Fig. 1). The stimulation that elicited the highest response in peak amplitude was determined and reduced to elicit approximately $30 \%-50 \%$ of the peak amplitude (pAmp) in order to control for ceiling effects.

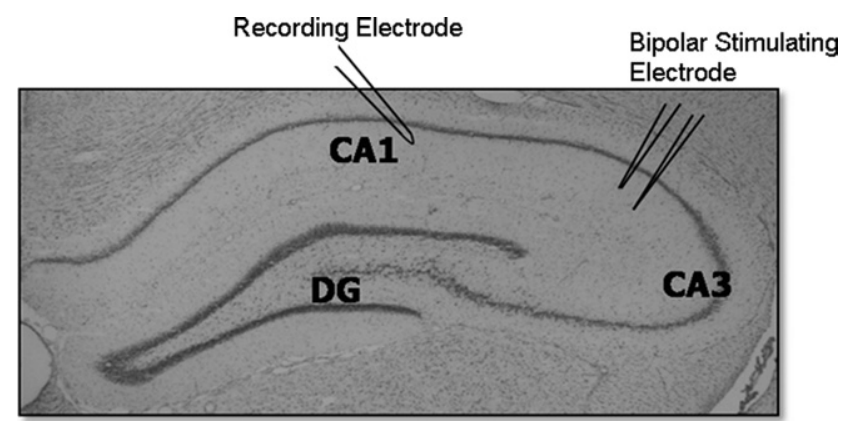

Fig. 1. Electrophysiological recordings on hippocampal slices. Anatomical section of hippocampus depicting the major subregions of the hippocampus and the positioning of electrodes for electrophysiological recordings. A bipolar stimulating electrode was positioned in area CA3 for the stimulation of the Schaffer collateral pathway and responses were recorded utilizing a recording electrode positioned in the CA1 region.

\subsubsection{Basal synaptic transmission}

Input/output (I/O) curves, curves depicting the response to a stepwise increase in stimulation intensity in $\mathrm{Y}-\mathrm{S}, \mathrm{O}-\mathrm{S}$, and $\mathrm{O}-\mathrm{GH}$, were used to examine the effect of chronic $\mathrm{GH}$ treatment on age-related changes in basal glutamatergic synaptic transmission. I/O curves were generated for compound (CMPD), as well as AMPA-R and NMDA-R mediated responses of neurons in the CA1 region following electrical stimulation of the Schaffer collaterals. Compound responses consisted of fEPSPs recorded under baseline conditions. AMPA-R mediated fEPSPs were recorded in the presence of APV and NMDA-R receptor fEPSPs were recorded in the presence of DNQX, bicuculline, and glycine. The amplitude of 3 averaged fEPSPs, elicited at an interpulse interval of 20 seconds utilizing the Axon Instruments (Foster City, CA, USA) pCLAMP 9.0 software, was plotted as a function of the logarithm exponent of stimulus intensity $\left(\log \mathrm{E}_{(\mathrm{STIM})}\right)$. Best-fit sigmoid curves were created to compare all 3 experimental groups under CMPD, AMPA-R, and NMDA-R recording conditions. The median effective stimulus intensity was determined from the best-fit sigmoid curve for each animal for CMPD, AMPA-R, and NMDA-R conditions. Because all curves reached a ceiling effect where the maximal response obtained was normalized for all experimental groups, the median effective stimulus intensity was used to assess differences between animal groups for all conditions. The median effective stimulus intensity was defined as the stimulus intensity that elicited $50 \%$ of the maximum obtainable pAmp.

\subsubsection{Paired-pulse ratios}

A paired-pulse facilitation assay was used to assess changes in glutamate release probability (Xu-Friedman and Regehr, 2004; Zucker and Regehr, 2002). Pairs of fEPSPs were evoked at an interpulse interval of $50 \mathrm{~ms}$ and pairedpulse ratios (PPRs) were calculated from the average of 5 pairs of fEPSPs by dividing the peak of the second fEPSP (P2) by that of the first (P1) evoked pulse.

\subsection{3. $L T P$}

Synaptic plasticity was assessed by examining changes in LTP in slices from Y-S, O-S, and O-GH rats. LTP was generated by using a high frequency stimulus train programmed through the Axon Instruments pCLAMP LTP Assistant by creating 2 subprotocols. The first subprotocol elicited pulses every 20 seconds at a stimulus intensity that generated approximately $30 \%-50 \%$ of the pAmp response for 10-15 minutes. Field EPSPs recorded under the first protocol were used to establish a pre-LTP baseline. When a stable baseline was recorded, which was defined as a response within $10 \%$ of the mean peak amplitude, the second subprotocol was utilized to generate a train of high-frequency stimulation (HFS; $100 \mathrm{~Hz}$ ) for 1 second in order to induce LTP. Recordings then were continued for 35 minutes during baseline stimulation with pulses every 20 seconds. LTP was defined as the sustained increase in potentiation 
lasting for at least 35 minutes. Hippocampal slices were discarded if the increase in potentiation was not sustained for 35 minutes following HFS. Percent potentiation was determined by comparing the last 5 minutes of potentiation to the last 5 minutes of baseline recordings pre-LTP.

\subsection{Western blotting}

For Western blot analysis of glutamate receptor subunit levels, Y-S, O-S, and O-GH rats ( $n=12$ per group) were anesthetized with sodium pentobarbital $(150 \mathrm{mg} / \mathrm{kg})$ and decapitated, the brains were removed, and the hippocampus dissected into subregions (Newton et al., 2005). Protein levels of subunits in the hippocampal subregion CA1 were determined in Western blot experiments as described previously (Shi et al., 2006, 2007). Briefly, tissue was homogenized using a Laemmli-based buffer supplemented with protease inhibitors and protein concentrations were determined using the bicinchoninic acid (BCA) method (Pierce Technology, Rockford, IL, USA). For analysis of NMDA-R and AMPA-R subunits levels, samples were loaded onto gels, separated using sodium dodecyl sulfate-polyacrylamide gel electrophoresis (SDS-PAGE), transferred onto membranes, and probed with the following primary antibodies: rabbit monoclonal antibody to the NR1 $(0.06 \mu \mathrm{g} / \mathrm{mL}$ Chemicon Billerica, MA, USA), NR2B $(0.07 \mu \mathrm{g} / \mathrm{mL}$ Chemicon), NR2A (0.07 $\mu \mathrm{g} / \mathrm{mL}$, Chemicon), GluR1 (0.01 $\mu \mathrm{g} / \mathrm{mL}$, Upstate, Placid, NY, USA), GluR2 (0.25 $\mu \mathrm{g} / \mathrm{mL}$, Chemicon), and mouse monoclonal antibody to actin (0.002 $\mu \mathrm{g} / \mathrm{mL}$, Chemicon). Peroxidase-conjugated donkey anti-rabbit IgG (10 ng/mL, Jackson ImmunoResearch Laboratories, West Grove, PA, USA) and peroxidaseconjugated donkey anti-mouse IgG (10 ng/mL, Jackson Laboratory) secondary antibodies were used with SuperSignal West Pico chemiluminescent Substrate (Pierce Technology) for detection. Blots were exposed on Kodak Biomax film (Sigma-Aldrich, St. Louis, MO, USA) and individual bands were quantified with Bio-Rad VersaDoc and Quantity one analysis software (Bio-Rad Laboratories, Hercules, CA, USA) in order to determine the relative amounts of each protein. The Gaussian trace optical density of each band was normalized to the average density of all of the matching bands within each cohort to permit comparison among blots and experiments. Each cohort represents 1 animal from each of the 3 different treatment groups and the matching bands are bands that represent 1 of the proteins, i.e., NR1. A repeated measurement was excluded if it was greater than 2 standard deviations away from the mean. Each blot was repeated at least once, thus generating a minimum of 4 acceptable measurements per antibody per sample. In each gel, actin was measured as internal loading control. We did not normalize our bands to these protein levels but looked for group differences in the actin levels. This method of quantifying protein levels in the subregions of the hippocampus has been used previously by our laboratory in multiple studies (Adams et al., 2008; Newton et al., 2008; Shi et al., 2006, 2007).

\subsection{Enzyme-linked immunosorbent assay (ELISA)}

IGF-I levels in plasma and hippocampus were determined as described previously (Adams et al., 2009). Briefly, $\mathrm{Y}-\mathrm{S}, \mathrm{O}-\mathrm{S}$, and O-GH rats ( $n=12$ per group) were anesthetized, cardiac blood was removed from the left ventricle of the heart, centrifuged, and plasma IGF-I levels (reported as mg IGF-I per mL plasma) were quantified using a modified ELISA ( $\mathrm{R} \& \mathrm{D}$ Systems Quantikine mouse IGF-I immunoassay; MG100; Minneapolis, MN, USA). After blood samples were removed, rats were perfused transcardially with phosphate-buffered saline containing $5 \mathrm{mM}$ dextrose and decapitated. The hippocampus was removed and homogenized in $1 \mathrm{M}$ acetate homogenization buffer for IGF-I acid extraction. IGF-I levels in hippocampus ( $\mu \mathrm{g}$ IGF-I per mg hippocampus) were determined using the same ELISA modification as for plasma.

\subsection{Statistics}

The median effective stimulus intensity for CMPD, AMPA-R, and NMDA-R dependent I/O curves were analyzed using a 1-way analysis of variance (ANOVA) followed by the Newman-Keuls post hoc test for pairwise comparisons when appropriate. Paired-pulse ratios of the second to the first evoked response were analyzed by a 1-way ANOVA followed by the Newman-Keuls post hoc test. For the analysis of LTP, the percentage potentiation above baseline in $\mathrm{Y}-\mathrm{S}, \mathrm{O}-\mathrm{S}$, and $\mathrm{O}-\mathrm{GH}$ was analyzed by a 1-way ANOVA followed by the Newman-Keuls post hoc test. The effect of $\mathrm{GH}$ on glutamatergic subunit proteins, was examined by comparing the normalized Gaussian trace optical densities for NR1, NR2A, NR2B, GluR1, GluR2, and actin using a 1-way ANOVA followed by the NewmanKeuls post hoc test. Finally, IGF-I levels in plasma and hippocampus for each experimental group were analyzed by a 1-way ANOVA followed by the Newman-Keuls post hoc test. Statistical significance was defined as $p<0.05$. All statistical analyses were done using SigmaStat 3.5 (Aspire Software International, Ashburne, VA, USA) software; GraphPad Prism 5 (GraphPad Software, Inc., San Diego, CA, USA) was used for curve fitting.

\section{Results}

\subsection{GH treatment alters AMPA- and NMDA-dependent basal synaptic transmission}

To determine the effect of 6-8 months of GH treatment on basal synaptic transmission in the CA1 region of old rats, the $\mathrm{I} / \mathrm{O}$ relationship of fEPSPs recorded in slices prepared from $\mathrm{Y}-\mathrm{S}, \mathrm{O}-\mathrm{S}$, and $\mathrm{O}-\mathrm{GH}$ rats was examined. Best-fit sigmoid curves were generated and the half maximal $\log \mathrm{E}_{\text {(STIM) }}\left(\log \mathrm{E}_{\text {(STIM1/2MAX) }}\right)$ was compared for all conditions. The results of the curve fitting analysis were similar 
among groups for CMPD fEPSPs and the half maximal $\log \mathrm{E}_{\text {(STIM) }}$ determined for the CMPD responses showed no group differences ( $p=0.997$, Fig. 2A). The results of the curve-fitting analysis for AMPA-R-dependent fEPSPs revealed that although there were no age-related changes in baseline responses, GH treatment enhanced AMPA-R synaptic transmission as evidenced by the significant decrease in the stimulation intensity required to evoke a half-maximal AMPA-R fEPSP response (Fig. 2B). The results of the curve-fitting analysis for NMDA-R-dependent fEPSPs demonstrated that a stronger stimulation intensity was required to elicit half-maximal responses in O-S rats compared with $\mathrm{Y}-\mathrm{S}$ rats, and this effect is reversed by GH treatment (Fig. 2C).

To determine whether chronic GH treatment altered glutamate release at excitatory synapses in the CA1 region, we measured PPRs in Y-S, O-S, and O-GH rats. PPR is the ratio between the second stimulus pulse $(\mathrm{P} 2)$ and the first stimulus pulse (P1) with an interpulse interval of $50 \mathrm{~ms}$. Changes in the PPR are often used as an indicator of alterations in neurotransmitter release probability, consistent with changes of presynaptic origin (Xu-Friedman and Regehr, 2004; Zucker and Regehr, 2002). The results indicate no difference among Y-S, O-S, and O-GH rats in PPRs recorded in hippocampal slices ( $p=0.662$; Fig. 3).

\subsection{GH treatment enhances long-term potentiation}

To assess the effect of GH treatment on synaptic plasticity we utilized an HFS protocol $(100 \mathrm{~Hz}$ per 1 second duration) to elicit LTP of CMPD CA1 region fEPSPs in hippocampal slices from Y-S, O-S, and O-GH rats. In slices from all 3 groups, HFS elicited a rapid increase in the amplitude of fEPSPs followed by a sustained potentiation, or LTP, that lasted for at least 30-35 minutes after induction (Fig. 4A). The average pAmp response of the last 5 minutes of sustained LTP was compared with the last 5 minutes of baseline recordings, and the percentage potentiation above baseline was derived. Hippocampal slices from $\mathrm{O}-\mathrm{GH}$ rats exhibited an enhanced potentiation compared with both Y-S and O-S rats (Fig. 4A). Importantly, analysis of the percentage potentiation indicated that LTP in O-GH rats was significantly enhanced compared with $\mathrm{O}-\mathrm{S}$ rats $(p=0.016$; Fig. 4B).

\subsection{Glutamate receptor subunit levels are unaltered by GH treatment}

In order to determine whether chronic GH treatment altered total protein levels of NMDA-R and AMPA-R subunits in the CA1 region, Western blot analysis was used to compare relative protein levels of those subunits in Y-S, $\mathrm{O}-\mathrm{S}$, and O-GH rats (Fig. 5). Our results demonstrated that there were no age-related differences in protein levels for NMDA-R subunits NR1 or NR2A (all $p$-values $>0.05$; Fig. $6)$. However, an age-related difference was detected for subunit NR2B ( $p<0.001$; Fig. 6). As with NMDA subunits
NR1 and NR2A, AMPA-R subunits and actin did not exhibit a difference in levels with age (all $p$-values $>0.05$; Fig. 6). In addition, all subunit and actin levels did not differ significantly between O-S and O-GH (all $p$-values $>0.05$; Fig. 6).

\subsection{IGF-I plasma and hippocampal levels are unaltered by $\mathrm{GH}$ treatment}

IGF-I levels in plasma and hippocampus were assessed to determine if those levels were altered by the chronic GH treatment in old rats (Fig. 7A and B). This is the same GH treatment that enhanced AMPA-R and NMDA-R dependent basal synaptic transmission and synaptic plasticity in CA1 region of old animals. Neither plasma nor hippocampal levels of IGF-I differed significantly between O-S and $\mathrm{O}-\mathrm{GH}$ rats (both $p$-values $>0.05$ ) or between $\mathrm{Y}-\mathrm{S}$ and $\mathrm{O}-\mathrm{S}$ (both $p$-values $>0.05$ ) rats; however, plasma IGF-I was significantly less in O-GH compared with Y-S rats $(p=$ 0.001; Fig. 7A). Furthermore, we observed a significant increase in body weight with age but not with treatment (data not shown; mean \pm standard error: Y-S $473 \pm 17.4 \mathrm{~g}$; O-S $585 \pm 18.5 \mathrm{~g}$; O-GH $623 \pm 16.4 \mathrm{~g}$; for Y-S both $p$-values $<0.005$ and for O-S and O-GH $p=0.564$ ).

\section{Discussion}

The results of this study provide the first evidence of an effect of chronic GH treatment on basal excitatory synaptic transmission and synaptic plasticity in the hippocampus. Six to 8 months of twice daily GH treatment enhanced AMPAR-dependent synaptic transmission and ameliorated the agerelated deficit in NMDA-R-dependent synaptic transmission in old Fisher $344 \times$ Brown Norway rats. In addition, GH treatment increased the magnitude of LTP, indicating enhanced synaptic plasticity. The absence of GH-induced changes in paired-pulse facilitation further suggests that the observed enhancement in synaptic transmission may not be mediated by presynaptic facilitation of glutamate release. In addition, the observed changes in synaptic transmission and plasticity following chronic GH treatment occurred in the absence of either alterations in levels of glutamate receptor subunits or elevations in plasma or hippocampal IGF-I levels in the CA1 region.

\subsection{Basal synaptic transmission}

Aging alters basal excitatory transmission in old rats. We observed a significant decrease in NMDA receptor function in old rats. These data are consistent with that from EcklesSmith et al. (2000) and Barnes et al. (1997). While we did observe an age-related decline in the NMDA receptor-mediated fEPSP in old rats, we saw no age-related change in the AMPA-R mediated fEPSP like that described by Barnes et al. (1997). The dissimilarity in the 2 results may result from the use of 2 different strains of rats. In the study by Barnes et al. (1997) F344 rats were used and in the present 



C

NMDA

\section{$\square$ Young Saline $\triangle$ Old Saline \\ O Old GH}

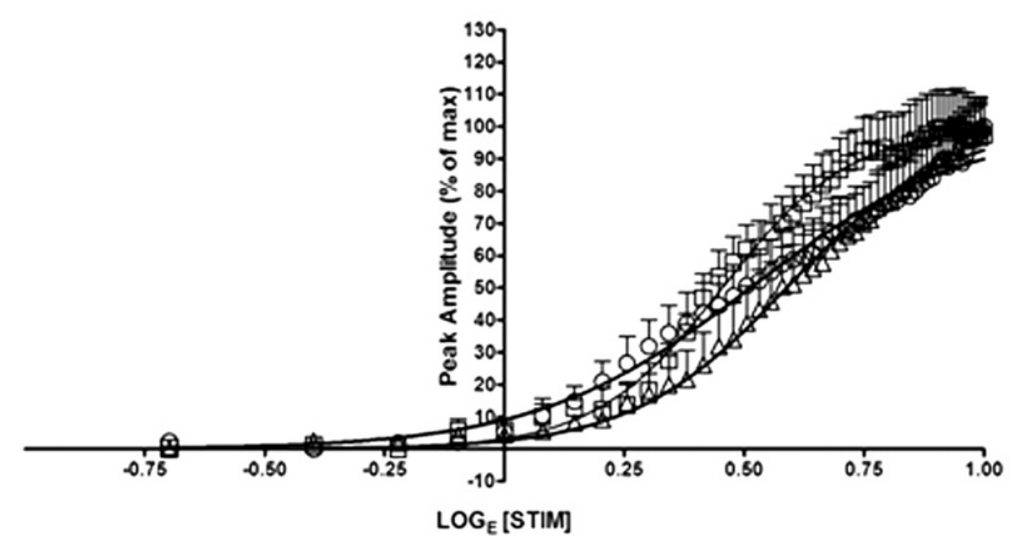



Fig. 2. Curve fitting analysis of compound (CMPD), $\alpha$-amino-3-hydroxy-5-methyl-4-isoxazole propionate (AMPA), and N-methyl-D-aspartate (NMDA) responses. Compound input/output (I/O) curves (A), AMPA receptor (R)-mediated I/O curves (B), and NMDA receptor (R)-mediated I/O curves (C) were generated from hippocampal CA1 field recordings. The stimulus intensity required to elicit the half-maximal response in each experimental group was compared as demonstrated by the bar graph. (A) Compound I/O curves were unaffected by age or growth hormone $(\mathrm{GH})$ treatment, $p=0.997$. 


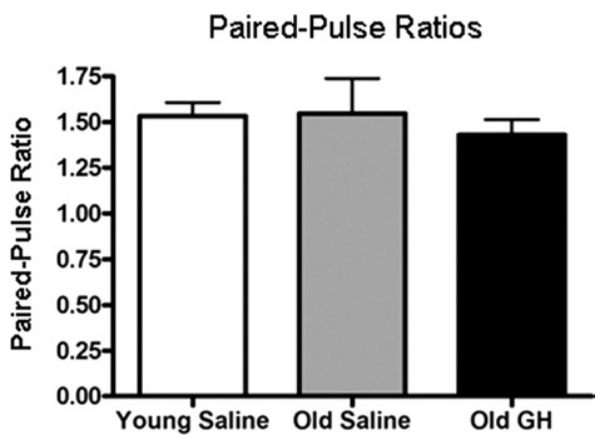

Fig. 3. Presynaptic facilitation. Bar graph illustrating the paired-pulse ratio (amplitude pulse 2/amplitude pulse 1, interpulse interval: $50 \mathrm{~ms}$ ) of field excitatory postsynaptic potentials (fEPSPs) recorded from young saline rats and in old rats treated with either saline or growth hormone $(\mathrm{GH})$. No group differences in this measure were observed, $p>0.05$.

experiments the hybrid strain of the F344 X Brown Norway animals was used. In addition, we observed no difference in the CMPD responses between young and old rats consistent with the idea that age-related changes in the hippocampus are very subtle. Interestingly, GH treatment significantly reversed the age-related decline in the NMDA receptormediated fEPSPs and enhanced the AMPA-R-mediated fEPSPs in old rats. Thus, GH alters the age-related course of excitatory transmission.

The data demonstrated subtle age-related changes in basal excitatory transmission. Compared with the young salinetreated rats, old saline-treated rats demonstrated a decline in NMDA-R-dependent, but not in AMPA-R-dependent fEPSPs. Our data revealed that this decline in synaptic transmission occurs in the absence of any alterations in the NMDA-R subunits NR1 and NR2A and AMPA-R subunits GluR1 and GluR2 tissue protein levels. However, consistent with a reduction in NMDA-R-dependent synaptic function the NMDA-R subunit NR2B is observed to decrease with age, and thus could contribute to the observed deficit in NMDA-R-dependent synaptic transmission. Although both AMPA-R and NMDA-Rdependent synaptic transmission were enhanced by chronic GH treatment, GH treatment did not lead to the alteration of protein levels of any of the subunits in the CA1 region. Nevertheless, an absence of a difference in subunit levels between old saline-treated and old GH-treated groups does not rule out an effect of GH on NMDA-R or AMPA-R subunits in the CA1 region. For example, subunit changes could occur within an individual layer in the CA1 region, but be masked by the stability of subunit protein levels in the other layers. Such a layer-specific change is seen in the age-related decline in synaptophysin levels that are limited to the stratum laconosum moleculare in the CA3 region (Smith et al., 2000). In addition, subunit changes limited to the postsynaptic density could be obscured by a stability of subunits in the cytosol as suggested by reports indicating that synaptic fractions reveal subtle changes in subunit expression levels that are not evident in the whole homogenate (Zhao et al., 2009).

\subsection{Long-term potentiation}

Our findings indicate that there was not a significant decline in LTP with age nevertheless a trend toward a decline was observed, and LTP was enhanced in GH-treated old rats compared with old saline-treated rats. Previous studies have demonstrated that there is a decline in LTP with age (Barnes et al., 1997; Eckles-Smith et al., 2000). The discrepancy between previous findings and our findings may be attributed to subtle differences in response to LTP protocols. A previous study demonstrated that, while LTP was generated robustly in young and old rats by multiple episodes of theta-burst stimulation and paired low frequency stimulation, no difference in the magnitude of LTP was observed (Kumar et al., 2007). Thus, while aging may impair LTP, this alteration may be masked by differences in protocols used to elicit LTP.

The HFS protocol used here to induce LTP is well-recognized to be dependent on NMDA-R function (Collingridge et al., 1983; Kimura et al., 1989; Morris et al., 1986). Interestingly, not only do our findings indicate that basal NMDA-R function was significantly increased by chronic GH treatment we also observed a significant enhancement in LTP following GH treatment in old rats. Acute GH treatment has also been observed to play a direct role in the enhancement of NMDAR-dependent basal synaptic transmission in juvenile (1.5-3month-old; Mahmoud and Grover, 2006), as well as young adult (10-month-old) and old (28-month-old) rats (Molina et al., unpublished observations). Furthermore, Mahmoud and Grover (2006) demonstrated that although GH induced a robust enhancement of synaptic transmission in hippocampal slices, this GH-induced enhancement could be prevented by eliciting LTP induced by HFS prior to GH application. Similarly a pretreatment of slices with GH prevented LTP induced by HFS. This finding suggests that GH shares at least some of the signaling mechanisms involved in tetanus-induced LTP, and thus, acute GH application can induce LTP and lead to LTP saturation preventing further potentiation (Castro et al., 1989; Mahmoud and Grover, 2006; McNaughton et al., 1986; Moser et al., 1998). One possible mechanism whereby GH could be influencing NMDA-R-dependent transmission is through the regulation of NMDA-R composition at the synapse. Specifically, changes in the ratio of NR1/NR2A and

(B) AMPA-R-mediated field excitatory postsynaptic potentials (fEPSPs) did not differ between young and old saline-treated rats, but slices from old GH-treated rats showed significant enhancement ( $p=0.01 ; *$ Old GH significantly different from young and old saline groups). (C) NMDA-R-mediated fEPSPs declined with age $p<0.001$, and these responses were enhanced by chronic GH treatment to a greater responsiveness than that observed in old $p=$ 0.011 and young saline rat groups, $p=0.008$ (* Old Saline significantly different from young saline and old GH groups). 


\section{A. LTP Time Course}


Fig. 4. Long-term potentiation (LTP). The time course of LTP induction in young saline rats and in old rats treated with saline or growth hormone (GH) is represented as percent amplitude of enhancement compared with baseline (A). Analysis of the percentage potentiation in the last 5 minutes of LTP (30-35 minutes) compared with the baseline (1-5 minutes) (B) for each treatment group revealed that LTP in old GH-treated rats was significantly greater than in old saline rats $p=0.016(*)$; young saline rats did not differ significantly from either old group.

NR1/N2RB receptor subunits which are reported to be associated with changes in LTP (Liu et al., 2004) could be occurring in $\mathrm{GH}$-treated rats that have stable cellular levels of individual subunits. For instance, long-term depression (LTD) but not LTP was abolished in hippocampal slices in which the proportion of the NR2B subunit was reduced by the selective NR2B blocker ifenprodil and the specific antagonist Ro256981. Furthermore, preferential inhibition of the NR2A subunit by the NR2A specific antagonist NVP-AAM077 prevented LTP (Liu et al., 2004).

Alterations in the proportions of NR2A and NR2B subunits in NMDA-R complexes also could alter membrane properties, particularly $\mathrm{Ca}^{2+}$ regulation which in turn could impact LTP. In addition the source of $\mathrm{Ca}^{2+}$, whether intracellular or extracellular, also plays a role in the direction of

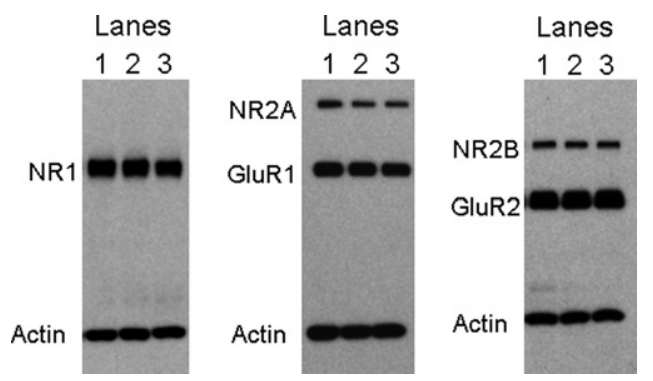

Fig. 5. Representative Western blots for synaptic proteins in the CA1 region of hippocampus. Western blot analysis was performed to assess CA1 protein levels of the N-methyl-D-aspartate (NMDA) subunits NR2A, NR1, and NR2B, and the alpha-amino-3-hydroxy-5-methyl-4-isoxazolepropionate (AMPA) subunits GluR1 and GluR2. Actin was assessed on each blot to insure equal protein loading. Experimental groups were: lane 1, young saline-treated; lane2, old saline-treated; and lane 3, old growth hormone $(\mathrm{GH})$-treated Fischer $344 \times$ Brown Norway rats. synaptic plasticity. For example, lowering the extracellular $\mathrm{Ca}^{2+}$ concentration in a slice medium during electrophysiological recordings can change a protocol that normally induces LTD to 1 that produces LTP in the hippocampal CA1 region (Mulkey and Malenka, 1992). In addition NR2B containing receptors are reduced in aged animals; this reduction has been demonstrated to reduce LTP and compromise learning and memory (Clayton et al., 2002). Thus, impairment in NMDA-R-dependent $\mathrm{Ca}^{2+}$ regulation with age could contribute to a reduction in LTP. For instance, a decrease in NR2B levels with age could be associated with a decrement in extracellular $\mathrm{Ca}^{2+}$ levels, which has been observed in aged rats (Audinat et al., 1994; Campbell et al., 1996; Clayton et al., 2002; Thibault et al., 1998; Vallano et al., 1996). Importantly, GH has been shown to increase cytoplasmic $\mathrm{Ca}^{2+}$ levels in BRIN-B11 cells, and this augmentation can be ablated by blocking elements of the GH signaling cascade (Zhang et al., 2006). Thus, the $\mathrm{GH}$-induced enhancement of LTP reported here could involve alterations to the subunit composition of NMDA-R at the postsynaptic membrane as well as GH-mediated changes to intracellular $\mathrm{Ca}^{2+}$ levels.

In addition to changes in the ratio of subunits comprising the NMDA-R, the properties of individual AMPA-R and NMDA-R subunits can be altered by posttranslational modifications such as phosphorylation. Phosphorylation can change the properties of receptor subunits and these changes can contribute to LTP enhancement. For instance, protein kinase $\mathrm{C}$ (PKC) and $\mathrm{Ca}^{2+}$ calmodulin kinase II (CaMKII) are known to phosphorylate glutamate receptor subunits NR1 and GluR1, respectively (Cammarota et al., 1998; Kim et al., 2008). Phosphorylation of these 2 subunits has been reported to underlie the enhancement in LTP that occurs 

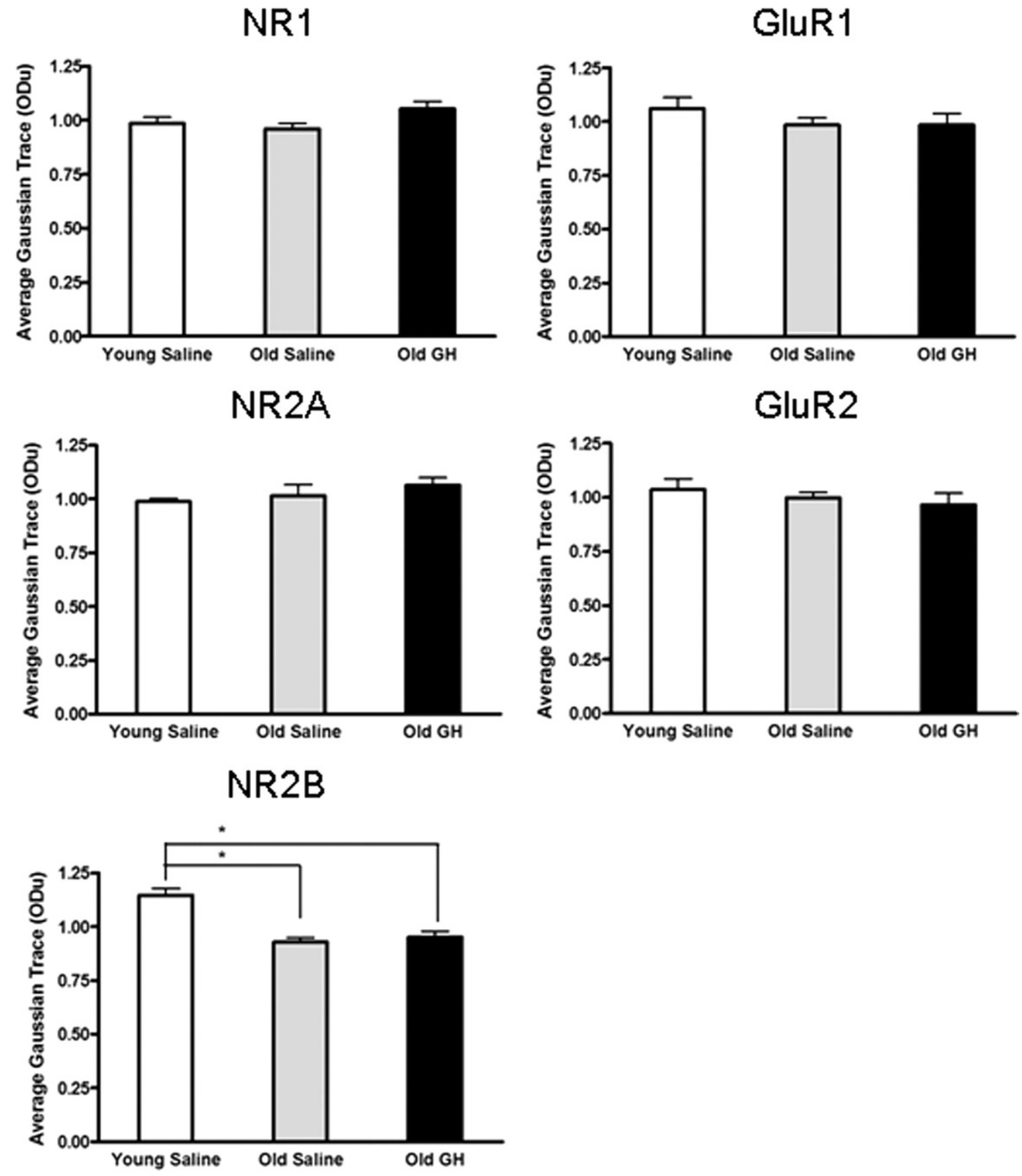

Fig. 6. Assessment of glutamate receptor subunit protein level changes. Densitometric Western blot analysis of subunit proteins in the CA1 region evaluated relative levels of the NR1, NR2A, and NR2B subunits of the N-methyl-D-aspartate (NMDA) receptor, and the GluR1 and GluR2 subunits of the alpha-amino-3-hydroxy-5-methyl-4-isoxazolepropionate (AMPA) receptor. The analysis revealed that only NR2B declined significantly with age $(p<$ 0.001 ), an effect that was not mitigated by growth hormone (GH) treatment (* Young saline significantly different from old saline and old GH groups). Chronic GH treatment twice daily for 6-8 months did not produce a significant change in the protein levels of any of the NMDA or AMPA subunits compared with that present in old saline-treated animals.

after treatment with nefiracetam, a nootropic drug reported to improve cognitive function and memory (Moriguchi et al., 2008). Thus, the GH-induced enhancement in LTP could be mediated by increasing the activation of kinases involved in the phosphorylation of NMDA and AMPA-R subunits.

\subsection{Insulin-like growth factor-I}

Considerable evidence in the literature suggests that IGF-I can serve as a mediator of the actions of GH, and the neurobiological effects of GH treatment are often attributed to a secondary increase in plasma levels of IGF-I (Florini 


\section{A Plasma IGF-I Levels}

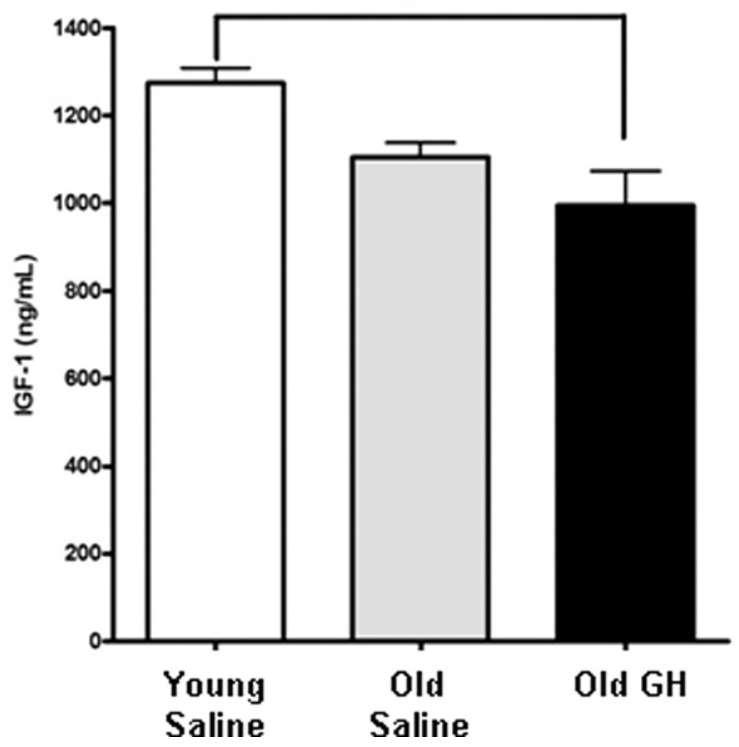

\section{B Hippocampal IGF-I Levels}



Fig. 7. Examination of insulin-like growth factor-I (IGF-I) plasma and tissue levels. IGF-I levels were determined in the plasma (A) and hippocampus (B) of young saline rats and old rats treated with either saline or growth hormone (GH). (A) The decrease in plasma IGF-I between young and old saline-treated rats fell short of significance $(p=0.098)$. Plasma IGF-I levels in old rats treated with GH do not differ significantly from old saline-treated rats, but were significantly lower than in young saline-treated rats $p=0.02$ (* Young saline-treated rats significantly different from old GH). (B) IGF-I levels in hippocampus did not differ with age or GH injection.

and Ewton, 1981; Sonntag et al., 1980). Consistent with this notion, treatment with GH and IGF-I have similar benefits for cognitive function in old animals (Markowska et al., 1998; Ramsey et al., 2004; Sonntag et al., 2000). In the present study, the data indicate that GH treatment resulted in enhanced synaptic transmission and plasticity in the absence of elevated IGF-I levels in either plasma or hippocampal tissue, suggesting a direct role for $\mathrm{GH}$ in the amelioration of age-related changes in synaptic function. Consistent with this possibility, recent studies have demonstrated that acute application of $\mathrm{GH}$ enhanced glutamatergic transmission in hippocampal slices from 1.5- to 3-month-old (Mahmoud and Grover, 2006) as well as from 10- and 28-month-old rats (Molina et al., unpublished observations). Importantly, $\mathrm{GH}$ receptors are expressed in the hippocampus as well as elsewhere in the brain (Burton et al., 1992; Fraser et al., 1990; Hull and Harvey, 1998; Lobie et al., 1993; Walsh et al., 1990; Zhai et al., 1994) and GH has been shown to cross the blood-brain barrier (Pan et al., 2005). Taken together, these studies and the present findings demonstrate that $\mathrm{GH}$ can act directly on the nervous system.

\subsection{Summary}

In summary, the present study demonstrates a direct role for $\mathrm{GH}$ in the mitigation of age-related changes in basal synaptic transmission and synaptic plasticity in the CA1 region of the hippocampus. This GH-mediated mitigation may contribute to the reported benefits of chronic GH treatment on cognitive impairment in old animals. Although the exact postsynaptic mechanism(s) through which $\mathrm{GH}$ enhances the activity of AMPA-R and NMDA-R will require additional study, they likely involve the regulation of receptor subunits at the postsynaptic membrane, either by increasing synaptic levels of the receptors or potentially altering their phosphorylation state. The changes in synaptic transmission and plasticity following chronic $\mathrm{GH}$ treatment reported here provide compelling evidence for a direct role for $\mathrm{GH}$ in the mediation of cellular events that are critical for the maintenance of optimal synaptic function. These data are consistent with the possibility that AMPA-R and NMDA-R provide potential targets for therapeutic interventions that could ultimately contribute to the enhancement of cognitive function in the elderly.

\section{Disclosure statement}

The authors disclose no conflicts of interest related to this work.

The animal protocol for this study conforms to National Institute of Health guidelines for the care and use of laboratory animals and was approved by the Animal Care and Use Committee of Wake Forest University Health Sciences.

\section{Acknowledgements}

The authors thank Dr. Ben Philpot for his helpful suggestions in the initial phases of recording long-term poten- 
tiation. This work was supported by NIH grants: NIA PO1 AG11370 and KO1 AG027828.

\section{References}

Aberg, D., 2010. Role of the growth hormone/insulin-like growth factor 1 axis in neurogenesis. Endocr. Dev. 17, 63-76.

Aberg, N.D., Brywe, K.G., Isgaard, J., 2006. Aspects of growth hormone and insulin-like growth factor-I related to neuroprotection, regeneration and functional plasticity in the adult brain. Sci. World J. 18, 53-80.

Aberg, N.D., Lind, J., Isgaard, J., George, K.H., 2010. Peripheral growth hormone induces cell proliferation in the intact adult rat brain. Growth Horm. IGF Res. 20, 264-269.

Adams, M.M., Forbes, E.M., Linville, C.M., Riddle, D.R., Sonntag, W.E., Brunso-Bechtold, J.K., 2009. Stability of local brain levels of insulinlike growth factor-I in two well-characterized models of decreased plasma IGF-I. Growth Factors 27, 181-188.

Adams, M.M., Shi, L., Linville, M.C., Forbes, M.E., Long, A.B., Bennett, C., Newton, I.G., Carter, C.S., Sonntag, W.E., Riddle, D.R., BrunsoBechtold, J.K., 2008. Caloric restriction and age affect synaptic proteins in hippocampal CA3 and spatial learning ability. Exp. Neurol. 211, 141-149.

Adams, M.M., Smith, T.D., Moga, D., Gallagher, M., Wang, Y., Wolfe, B.B., Rapp, P.R., Morrison, J.H., 2001. Hippocampal dependent learning ability correlates with $N$-methyl-D-aspartate (NMDA) receptor levels in CA3 neurons of young and aged rats. J. Comp. Neurol. 432, $230-243$.

Audinat, E., Lambolez, B., Rossier, J., Crépel, F., 1994. Activity-dependent regulation of N-methyl-D-aspartate receptor subunit expression in rat cerebellar granule cells. Eur. J. Neurosci. 6, 1792-1800.

Barnes, C.A., Rao, G., Shen, J., 1997. Age-related decrease in the Nmethyl-D-aspartateR-mediated excitatory postsynaptic potential in hippocampal region CA1. Neurobiol. Aging 18, 445-452.

Bliss, T.V., Lomo, T., 1973. Long-lasting potentiation of synaptic transmission in the dentate area of the anaesthetized rabbit following stimulation of the perforant path. J. Physiol. 232, 331-356.

Burton, K.A., Kabigting, E.B., Clifton, D.K., Steiner, R.A., 1992. Growth hormone receptor messenger ribonucleic acid distribution in the adult male rat brain and its colocalization in hypothalamic somatostatin neurons. Endocrinology 131, 958-963.

Cammarota, M., Bernabeu, R., Levi De Stein, M., Izquierdo, I., Medina, J.H., 1998. Learning-specific, time-dependent increases in hippocampal $\mathrm{Ca}^{2+}$ /calmodulin-dependent protein kinase II activity and AMPA GluR1 subunit immunoreactivity. Eur. J. Neurosci. 10, 2669-2676.

Campbell, L.W., Hao, S.Y., Thibault, O., Blalock, E.M., Landfield, P.W., 1996. Aging changes in voltage-gated calcium currents in hippocampal CA1 neurons. J. Neurosci. 16, 6286-6295.

Cao, X., Cui, Z., Feng, R., Tang, Y.P., Qin, Z., Mei, B., Tsien, J.Z., 2007. Maintenance of superior learning and memory function in NR2B transgenic mice during ageing. Eur. J. Neurosci. 25, 1815-1822.

Castro, C.A., Silbert, L.H., McNaughton, B.L., Barnes, C.A., 1989. Recovery of spatial learning deficits after decay of electrically induced synaptic enhancement in the hippocampus. Nature 342, 545-548.

Clayton, D.A., Mesches, M.H., Alvarez, E., Bickford, P.C., Browning, M.D., 2002. A hippocampal NR2B deficit can mimic age-related changes in long-term potentiation and spatial learning in the Fischer 344 rat. J. Neurosci. 22, 3628-3637.

Collingridge, G.L., Kehl, S.J., McLennan, H., 1983. Excitatory amino acids in synaptic transmission in the Schaffer collateral-commissural pathway of the rat hippocampus. J. Physiol. 334, 33-46.

Eckles-Smith, K., Clayton, D., Bickford, P., Browning, M.D., 2000. Caloric restriction prevents age-related deficits in LTP and in NMDA receptor expression. Brain Res. Mol. Brain Res. 78, 154-162.
Fischer, W., Gage, F.H., Björklund, A., 1989. Degenerative changes in forebrain cholinergic nuclei correlate with cognitive impairments in aged rats. Eur. J. Neurosci. 1, 34-45.

Florini, J.R., Ewton, D.Z., 1981. Insulin acts as a somatomedin analog in stimulating myoblast growth in serum-free medium. In Vitro 17, 763768.

Fraser, R.A., Attardo, D., Harvey, S., 1990. Growth hormone receptors in hypothalamic and extra-hypothalamic tissues. J. Mol. Endocrinol. 5, 231-238.

Frick, K.M., Baxter, M.G., Markowska, A.L., Olton, D.S., Price, D.L., 1995. Age-related spatial reference and working memory deficits assessed in the water maze. Neurobiol. Aging 16, 149-160.

Granger, R., Deadwyler, S., Davis, M., Moskovitz, B., Kessler, M., Rogers, G., Lynch, G., 1996. Facilitation of glutamate receptors reverses an age-associated memory impairment in rats. Synapse 22, 332-337.

Hollmann, M., Heinemann, S., 1994. Cloned glutamate receptors. Annu. Rev. Neurosci. 17, 31-108.

Hull, K.L., Harvey, S., 1998. Autoregulation of growth hormone receptor and growth hormone binding protein transcripts in brain and peripheral tissues of the rat. Growth Horm. IGF Res. 8, 167-173.

Isgaard, J., Aberg, D., Nilsson, M., 2007. Protective and regenerative effects of the GH/IGF-I axis on the brain. Minerva Endocrinol. 32, 103-113.

Kim, H.W., Roh, D.H., Yoon, S.Y., Seo, H.S., Kwon, Y.B., Han, H.J., Kim, K.W., Beitz, A.J., Lee, J.H., 2008. Activation of the spinal sigma-1 receptor enhances NMDA-induced pain via PKC- and PKAdependent phosphorylation of the NR1 subunit in mice. Br. J. Pharmacol. 154, 1125-1134.

Kimura, F., Nishigori, A., Shirokawa, T., Tsumoto, T., 1989. Long-term potentiation and $N$-methyl-D-aspartate receptors in the visual cortex of young rats. J. Physiol. 414, 125-144.

Kumar, A., Thinschmidt, J.S., Foster, T.C., King, M.A., 2007. Aging effects on the limits and stability of long-term synaptic potentiation and depression in rat hippocampal area CA1. J. Neurophysiol. 98, 594601.

Le Grevès, M., Steensland, P., Le Grevès, P., Nyberg, F., 2002. Growth hormone induces age-dependent alteration in the expression of hippocampal growth hormone receptor and $\mathrm{N}$-methyl-D-aspartate receptor subunits gene transcripts in male rats. Proc. Natl. Acad. Sci. U. S. A. 99, 7119-7123.

Liu, L., Wong, T.P., Pozza, M.F., Lingenhoehl, K., Wang, Y., Sheng, M., Auberson, Y.P., Wang, Y.T., 2004. Role of NMDA receptor subtypes in governing the direction of hippocampal synaptic plasticity. Science 304, 1021-1024.

Lobie, P.E., García-Aragón, J., Lincoln, D.T., Barnard, R., Wilcox, J.N., Waters, M.J., 1993. Localization and ontogeny of growth hormone receptor gene expression in the central nervous system. Brain Res. Dev. Brain Res. 74, 225-233.

Lobie, P.E., Zhu, T., Graichen, R., Goh, E.L., 2000. Growth hormone, insulin-like growth factor I and CNS: localization, function and mechanisms of action. Growth Horm. IGF Res. (suppl B), S51-S56.

Lynch, G., Rex, C.S., Gall, C.M., 2006. Synaptic plasticity in early aging. Ageing Res. Rev. 5, 255-280.

Magnusson, K.R., 1998. Aging of glutamate receptors: correlations between binding and spatial memory performance in mice. Mech. Ageing Dev. 104, 227-248.

Mahmoud, G.S., Grover, L.M., 2006. Growth hormone enhances excitatory synaptic transmission in area CA1 of rat hippocampus. J. Neurophysiol. 95, 2962-2974.

Markowska, A.L., Mooney, M., Sonntag, W.E., 1998. Insulin-like growth factor-1 ameliorates age-related behavioral deficits. Neuroscience 87 , $559-569$.

McNaughton, B.L., Barnes, C.A., Rao, G., Baldwin, J., Rasmussen, M., 1986. Long-term enhancement of hippocampal synaptic transmission and the acquisition of spatial information. J. Neurosci. 6, 563-571. 
Moriguchi, S., Shioda, N., Han, F., Narahashi, T., Fukunaga, K., 2008. $\mathrm{CaM}$ kinase II and protein kinase $\mathrm{C}$ activations mediate enhancement of long-term potentiation by nefiracetam in the rat hippocampal CA1 region. J. Neurochem. 106, 1092-1103.

Morris, R.G., Anderson, E., Lynch, G.S., Baudry, M., 1986. Selective impairment of learning and blockade of long-term potentiation by an $N$-methyl-D-aspartate receptor antagonist, AP5. Nature 319, 774-776.

Moser, E.I., Krobert, K.A., Moser, M.B., Morris, R.G., 1998. Impaired spatial learning after saturation of long-term potentiation. Science 281, $2038-2042$.

Mulkey, R.M., Malenka, R.C., 1992. Mechanisms underlying induction of homosynaptic long-term depression in area CA1 of the hippocampus. Neuron 9, 967-975.

Newton, I.G., Forbes, M.E., Legault, C., Johnson, J.E., Brunso-Bechtold, J.K., Riddle, D.R., 2005. Caloric restriction does not reverse agingrelated changes in hippocampal BDNF. Neurobiol. Aging 26, 683-688.

Newton, I.G., Forbes, M.E., Linville, M.C., Pang, H., Tucker, E.W., Riddle, D.R., Brunso-Bechtold, J.K., 2008. Effects of aging and caloric restriction on dentate gyrus synapses and glutamate receptor subunits. Neurobiol. Aging 29, 1308-1318.

Olton, D.S., Werz, M.A., 1978. Hippocampal function and behavior: Spatial discrimination and response inhibition. Physiol. Behav. 20, 597605.

Pan, W., Yu, Y., Cain, C.M., Nyberg, F., Couraud, P.O., Kastin, A.J., 2005. Permeation of growth hormone across the blood-brain barrier. Endocrinology 146, 4898-4904.

Ramsey, M.M., Adams, M.M., Ariwodola, O.J., Sonntag, W.E., Weiner, J.L., 2005. Functional characterization of des-IGF-1 action at excitatory synapses in the CA1 region of rat hippocampus. J. Neurophysiol. 94, 247-254.

Ramsey, M.M., Weiner, J.L., Moore, T.P., Carter, C.S., Sonntag, W.E., 2004. Growth hormone treatment attenuates age-related changes in hippocampal short-term plasticity and spatial learning. Neuroscience 129, 119-127.

Rapp, P.R., Rosenberg, R.A., Gallagher, M., 1987. An evaluation of spatial information processing in aged rats. Behav. Neurosci. 101, 3-12.

Rosenzweig, E.S., Rao, G., McNaughton, B.L., Barnes, C.A., 1997. Role of temporal summation in age-related long-term potentiation-induction deficits. Hippocampus 7, 549-558.

Shi, L., Adams, M.M., Linville, M.C., Newton, I.G., Forbes, M.E., Long, A.B., Riddle, D.R., Brunso-Bechtold, J.K., 2007. Caloric restriction eliminates the aging-related decline in NMDA and AMPA receptor subunits in the rat hippocampus and induces homeostasis. Exp. Neurol. 206, 70-79.

Shi, L., Adams, M.M., Long, A., Carter, C.C., Bennett, C., Sonntag, W.E., Nicolle, M.M., Robbins, M., D’Agostino, R., Brunso-Bechtold, J.K., 2006. Spatial learning and memory deficits after whole-brain irradiation are associated with changes in NMDA receptor subunits in the hippocampus. Radiat. Res. 166, 892-899.

Smith, T.D., Adams, M.M., Gallagher, M., Morrison, J.H., Rapp, P.R., 2000. Circuit-specific alterations in hippocampal synaptophysin immu- noreactivity predict spatial learning impairment in aged rats. J. Neurosci. 2000, 6587-6593.

Sonntag, W.E., Bennett, S.A., Khan, A.S., Thornton, P.L., Xu, X., Ingram, R.L., Brunso-Bechtold, J.K., 2000. Age and insulin-like growth factor-1 modulate N-methyl-D-aspartate receptor subtype expression in rats. Brain Res. Bull. 51, 331-338.

Sonntag, W.E., Steger, R.W., Forman, L.J., Meites, J., 1980. Decreased pulsatile release of growth hormone in old male rats. Endocrinology 107, 547-554.

Tang, Y.P., Shimizu, E., Dube, G.R., Rampon, C., Kerchner, G.A., Zhuo, M., Liu, G., Tsien, J.Z., 1999. Genetic enhancement of learning and memory in mice. Nature 401, 63-69.

Thibault, O., Porter, N.M., Chen, K.C., Blalock, E.M., Kaminker, P.G., Clodfelter, G.V., Brewer, L.D., Landfield, P.W., 1998. Calcium dysregulation in neuronal aging and Alzheimer's disease: history and new directions. Cell Calcium 24, 417-433.

Trejo, J.L., Piriz, J., Llorens-Martin, M.V., Fernandez, A.M., Bolós, M., LeRoith, D., Nuñez, A., Torres-Aleman, I., 2007. Central actions of liver-derived insulin-like growth factor I underlying its pro-cognitive effects. Mol. Psychiatry 12, 1118-1128.

Tsien, J.Z., Huerta, P.T., Tonegawa, S., 1996. The essential role of hippocampal CA1 NMDA receptor-dependent synaptic plasticity in spatial memory. Cell 87, 1327-1338.

Vallano, M.L., Lambolez, B., Audinat, E., Rossier, J., 1996. Neuronal activity differentially regulates NMDA receptor subunit expression in cerebellar granule cells. J. Neurosci. 16, 631-639.

Walsh, R.J., Mangurian, L.P., Posner, B.I., 1990. The distribution of lactogen receptors in the mammalian hypothalamus: an in vitro autoradiographic analysis of the rabbit and rat. Brain Res. 530, 1-11.

Wang, Y.T., Linden, D.J., 2000. Expression of cerebellar long-term depression requires postsynaptic clathrin-mediated endocytosis. Neuron 25, 635-647.

Woodruff-Pak, D.S., Foy, M.R., Akopian, G.G., Lee, K.H., Zach, J., Nguyen, K.P., Comalli, D.M., Kennard, J.A., Agelan, A., Thompson, R.F., 2010. Differential effects and rates of normal aging in cerebellum and hippocampus. Proc. Natl. Acad. Sci. U. S. A. 107, 1627-1629.

Xu-Friedman, M.A., Regehr, W.G., 2004. Structural contributions to shortterm synaptic plasticity. Physiol. Rev. 84, 69-85.

Zhai, Q., Lai, Z., Roos, P., Nyberg, F., 1994. Characterization of growth hormone binding sites in rat brain. Acta Paediatr. Suppl. 406, 92-95.

Zhang, F., Zhang, Q., Tengholm, A., Sjöholm, A., 2006. Involvement of JAK2 and Src kinase tyrosine phosphorylation in human growth hormone-stimulated increases in cytosolic free $\mathrm{Ca} 2+$ and insulin secretion. Am. J. Physiol. Cell Physiol. 291, C466-C475.

Zhao, X., Sun, L., Jia, H., Meng, Q., Wu, S., Li, N., He, S., 2009. Isolation rearing induces social and emotional function abnormalities and alters glutamate and neurodevelopment-related gene expression in rats. Prog. Neuropsychopharmacol. Biol. Psychiatry 33, 1173-1177.

Zucker, R.S., Regehr, W.G., 2002. Short-term synaptic plasticity. Annu. Rev. Physiol. 64, 355-405. 